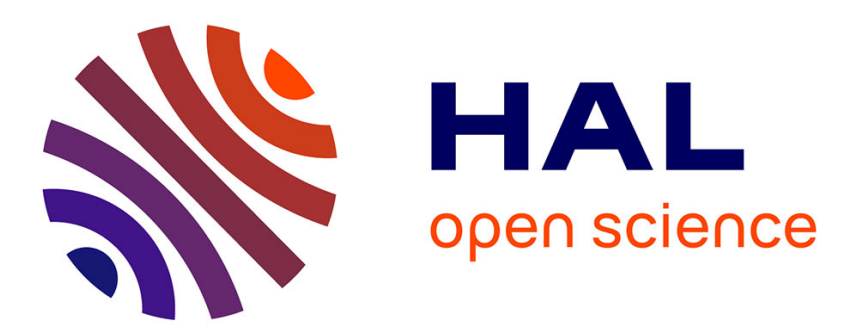

\title{
Control of a Piezoelectric Actuator Using a Bounded-Adaptive Backstepping Scheme and Sliding-Mode Observer
}

Juan Antonio Escareno, Didace Habineza, Micky Rakotondrabe

\section{To cite this version:}

Juan Antonio Escareno, Didace Habineza, Micky Rakotondrabe. Control of a Piezoelectric Actuator Using a Bounded-Adaptive Backstepping Scheme and Sliding-Mode Observer. The 19th World Congress of the International Federation of Automatic Control, IFAC'14, 2014, Cape Town, South Africa. pp.6556 - 6562. hal-01324790

\section{HAL Id: hal-01324790 \\ https://hal.science/hal-01324790}

Submitted on 1 Jun 2016

HAL is a multi-disciplinary open access archive for the deposit and dissemination of scientific research documents, whether they are published or not. The documents may come from teaching and research institutions in France or abroad, or from public or private research centers.
L'archive ouverte pluridisciplinaire HAL, est destinée au dépôt et à la diffusion de documents scientifiques de niveau recherche, publiés ou non, émanant des établissements d'enseignement et de recherche français ou étrangers, des laboratoires publics ou privés. 


\title{
Control of a Piezoelectric Actuator Using a Bounded-Adaptive Backstepping Scheme and Sliding-Mode Observer
}

\author{
${ }^{1}$ Juan ESCARENO, ${ }^{2}$ Didace HABINEZA \\ and ${ }^{2}$ Micky RAKOTONDRABE \\ ${ }^{1}$ Polytechnic Institute of Advanced Sciences 7-9 rue Maurice \\ Grandcoing, 94200 Ivry sur Seine, France \\ ${ }^{2}$ FEMTO-ST Institute, UMR CNRS - UFC / ENSMM / UTBM, \\ Automatic Control and Micro-Mechatronic Deparment, 24, rue Alain \\ Savary, Besançon, France. \\ e-mails:escareno@ipsa.fr; \{didace.habineza; mrakoton\}@femto-st.fr
}

\begin{abstract}
The present paper deals with the motion control of a piezoelectric cantilevered actuator. The motion behavior of the piezoelectric cantilever is affected by two main parasitic effects, the hysteresis and creep effects, which are considered as a generalized disturbance. Additionally, cantilevers's position is the only available measured state. The latter operational scenario reveals that the trajectory-tracking or regulation problem is a non-obvious task. Prior to the controller design, we have focused on the estimation of the velocity using a sliding-mode observer (SMO) in order to avoid the numerical derivative. On the other hand, the control design takes into account the limited response of the control input (bounded states) and it is obtained using the Backstepping framework assuring the stability of origin. Numerical simulations are first presented to verify the efficiency of the observer and controller algorithm, showing that the control objective is fulfilled. The experimental tests were carried out to validate and evaluated the control strategy.
\end{abstract}

Keywords: Piezoactuator Control, Sliding-mode Observer, Backstepping, Real-Time control

\section{INTRODUCTION}

The significant advance of microrobotics in recent years, from technological and theoretical point of view, has increased the application range in many fields. In the biomedical domain, the manipulation of biological agents (red blood cells or DNA) relies on piezoelectric-based micro-grippers. In the field of micro-assembly, a key application is the development of MEMS (Micro ElectroMechanical Systems) which themselves impact other areas as automotive, aeronautics and aerospace.

The attractive properties of piezoelectric actuators, with cantilevered structure in this case (called piezocantilever), which are the high resolution, the significant stiffness, and the large bandwidth exhibit great potential for applications like the manipulation of micro-sized objects. However, these active materials are constrained by adverse effects which are the hysteresis and the creep. Furthermore, it is important to underline that control systems, in practice, feature an amplitude-limited response. This aspect must be considered in the control design for stability and performance purposes. Hence, it is necessary

\footnotetext{
* This work is supported by the national ANR-Emergence MYMESYS-project (ANR-11-EMMA-006: High Performances Embedded Measurement Systems for multiDegrees of Freedom Microsystems). This work is also supported by the CNRS-project MIMHAC.
}

to develop appropriate control designs that encompasses disturbance rejection and bounded control inputs.

Controlling systems featuring hysteresis have been addressed using feedforward and feedback control strategies, or a combination of both. The former relies on different models to compensate for the adverse effects of the hysteresis, while the latter use robust/adaptive techniques that disregard the knowledge of the hysteresis model.

In feedforward control of piezoelectric actuators, several approaches are available to model and then to compensate for the hysteresis: the Preisach [1][2][3] and the PrandtlIshlinskii approaches [4][5][6]. In both, a complex hysteresis is modeled by the sum of many basic hysteresis (hysterons). Both approaches can be very accurate subject to the use of a high number of elementary hysteresis, which represents a computational burden implementation. Alternatively, the Bouc-Wen model of hysteresis, initially proposed by Bouc [7] and further modified by Wen [8], has an interesting simplicity and is able to represent a large class of hysteresis. Although the low cost and the high packageability (no sensors required) of the used feedforward control approaches, their main limitation is the lack of robustness face to model uncertainties and to external disturbances.

Concerning feedback control, several approaches have been used. They include linear robust control techniques based on $H_{\infty}$ [9] [10], sliding mode control (SMC) and adap- 
tive schemes [11][12][13][14] and state-feedback techniques [15][16]. Since the only measurement available in piezoelectric cantilevered actuator is the displacement (deflection of the cantilever), state-feedback control techniques required the use of observers. For this aim, [15] proposed a Kalman filtering computed to estimate non-measured states as well as for noise reduction in the measurement. In [17] an inverse-dynamics-based unknown input observer (UIO) was proposed to estimate the state vector as well as the force, considered as the external unknown input (disturbance). While the first observer was limited to linear model and then did not account for the hysteresis and creep of the actuator, the second observer was typified by a complex implementation. Furthermore, an essential point that is not considered by the above works is the bounded input control of the actuator. Indeed, if the voltage amplitude is too high, there is a risk of depolarization of the material leading to a loss of the piezoelectric properties. Consequently, overvoltages due to an excessive sollicitation on the piezoelectric actuator to effectuate rapid responses (large bandwidth) or large courses may destroy the latter.

The actual paper focuses on the motion control problem of a piezoelectric cantilever actuator (piezocantilever) operating under different references trajectories. The herein designed controller takes into account the fact that the system features a bounded-disturbed input. Also, given the fact that the only measured state is the piezocantilever's displacement, a sliding mode observer is employed to estimate the state vector and then used in the feedback control to ensure certain given performances for the closed-loop actuator. In the literature, several nonlinear tools have been introduced for analyzing and controlling linear and nonlinear systems with bounded inputs [22][23][24][27]. The control strategy presented in [27] has inspired us to propose a control strategy based on the backstepping technique because this considers not only bounded feedbacks but also bounded virtual controllers. More precisely, the developed controller considers the boundedness of the control inputs and it results in a simple and explicit expressions which are suitable for implementation. The proposed observer/control system is first verified by simulation which validates its efficiency. Then, an application to a home-made piezoelectric actuator with cantilevered structure was carried out and the experimental results validated the proposed approach.

The remainder of the paper is as follows. The description and model of the piezocantilever is presented in section2. In section-3, the disturbance observer based on the UIO Technique as well as the control strategy to solve the tracking problem of the piezocantilever motion are detailed. Simulation results are presented in section- 4 and experimental tests in section-5. Finally, the conclusions and perspectives are given in section- 6 .

\section{MODEL OF THE PIEZOCANTILEVER}

Without loss of generality relative to the modeling and control of piezoelectric actuators, the experimental test we will do in this paper and presented in section-5 is based on a piezocantilever. Piezocantilevers are largely used in the development of microgripper and micromanipulators in microassembly, micromanipulation and microrobotics

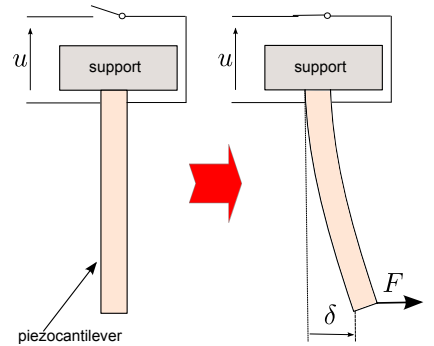

Fig. 1. (a) and (b): principle of a piezocantilevered actuator.

applications [18]. A piezocantilever, pictured in Fig.1-a, is a cantilever composed of two or several layers where at least one layer is based on piezoelectric material (piezolayer). The non-piezoelectric layers, called passive layer, is often a metal (copper, chrome...). When a voltage $u$ is applied to the piezolayer, it contracts or expands. Due to the interface constrain between the different layers and the difference on their contraction/elongation, the whole cantilever performs a bending $\delta$ (Fig.1-b). In the figure, $F$ is an external force applied to the piezocantilever if this latter is in interaction with the environment (objects...).

The dynamic and nonlinear behavior of a piezocantilever can be approximated by a second-order system, written as [19]:

$$
a \ddot{\delta}+b \dot{\delta}+\delta=d_{p} u-h+c
$$

where $h$ and $c$ denotes the hysteresis and creep contributions respectively, while $a, b$ and $d_{p}$ are positive coefficients obtained from an identification process.

In the model (1), the hysteresis contribution $h(t)$ has been modelled with the quadrilateral technique [19], the BoucWen equation [20], the Prandtl-Ishlinskii [21] or other techniques. This model (1) follows the Hammerstein structure which consists in cascading a linear dynamics with a nonlinear static part. Such structure is widely used to the modeling of hysteresis in piezoelectric systems. The creep contribution $c(t)$ has been considered as virtual external disturbance in [19], modelled with a low bandwidth linear dynamics in [21], and not accounted in many works.

The approach used in this paper consists in taking the hysteresis and creep as a single time-varying disturbance $\theta(t)=-h(t)+c(t)$. Furthermore, considering that the input voltage $u(t)$ should be limited in order to avoid the depolarization of the piezoelectric material, let us introduce the saturation operator for the control input of the actuator:

$$
\sigma(u(t))=\left\{\begin{array}{l}
+U ; \quad \text { for } u(t)>+U \\
u(t) ; \quad \text { for }-U \leq u(t) \leq+U \\
-U ; \quad \text { for } u(t)<-U
\end{array}\right.
$$

where $U>0$ is the saturation value. Therefore, the model (1) becomes:

$$
a \ddot{\delta}+b \dot{\delta}+\delta=d_{p} \sigma(u(t))+\theta
$$

In general, there is no prior information about the disturbance dynamics, hence it is logical to suppose first that

$$
\dot{\theta} \approx 0
$$

(4) implies that the disturbance is a slow time-varying with respect to estimation provided by the adaptive control law. Although, we will illustrate by simulation that the 
adaptive estimation is able to track some fast time-varying disturbances.

\section{CONTROL STRATEGY}

The problem addressed in this paper consists in fulfilling the tracking control objective of the piezoelectric actuator (for different trajectories and at different operational motion rates) with only the position as available measurement and with a saturated control input affected by an unknown-bounded disturbance (hysteresis and creep). However, to implement an efficient tracking controller, it is required to have a twice-differentiable reference vector $x^{d}=\left(\delta^{d}, \dot{\delta}^{d}\right)$, a full knowledge of the state vector $x=(\delta, \dot{\delta})^{T}$ and, if it is possible, the knowledge of the disturbance term. For these aims, we first study an observer based on the sliding mode. This permits to estimate the states without having numerical differentiation. Then, a backstepping control scheme is proposed. This ensures a robustness of the closed-loop although the uncertainties on the disturbance term (related to the hysteresis and creep nonlinearities) and although the saturated input control.

\subsection{Sliding-Mode Observer}

The modified super-twisting observer scheme is used to reconstruct the state vector [25]. Such observer has several appealing features: (i) the observer can be designed separately from the controller, (ii) it is insensitive to unknown inputs, and (iii) it has a finite-time to converge to the state-vector.

The form of the observer is given by

$$
\begin{aligned}
& \hat{\dot{x}}_{1}=\hat{x}_{2}+z_{1} \\
& \hat{\dot{x}}_{2}=f\left(t, x_{1}, \hat{x}_{2}, u\right)+z_{2}
\end{aligned}
$$

where

$$
\begin{aligned}
& z_{1}=\beta\left|x_{1}-\hat{x}_{1}\right|^{\frac{1}{2}} \operatorname{sign}\left(x_{1}-\hat{x}_{1}\right) \\
& z_{2}=\alpha \operatorname{sign}\left(x_{1}-\hat{x}_{1}\right)
\end{aligned}
$$

for our case $x_{1}=\delta, x_{2}=\dot{\delta}$ and

$$
f\left(t, x_{1}, \hat{x}_{2}, u\right)=\frac{1}{a}\left(-b \hat{x}_{2}-x_{1}+d_{p} u(t)\right)
$$

$\alpha>0$ and $\beta>0$ are the observer gains.

\subsection{Bounded-Adaptive Backsteppig}

The control objective is to design an amplitude-limited control to solve the tracking problem of the piezoelectric actuator, as well as to provide the corresponding stability analysis. To this end, the Backstepping technique provides an appropriate framework. Furthermore, notice that the aforementioned dynamic model (3) features a cascade form (lower-triangular) suitable to apply the Backstepping technique. Remind the model by assuming that the voltage $u(t)$ is within the limitation:

$$
a \ddot{\delta}+b \dot{\delta}+\delta=d_{p} u(t)+\theta
$$

Since we are concerned on solving the trajectory tracking problem, the latter differential model is rewritten into state-space model about the error variable $\xi$ :

$$
\begin{aligned}
& \dot{\xi}_{1}=\xi_{2} \\
& \dot{\xi}_{2}=\frac{1}{a}\left[d_{p} u(t)+\theta-b \dot{\delta}-\delta\right]-\ddot{\delta^{d}}
\end{aligned}
$$

where $\xi_{1}=\delta-\delta_{d}$ and $\xi_{2}=\dot{\delta}-\dot{\delta}_{d}$ are the position and velocity errors, respectively.

For the sake of ease of writing, let us use the following notation simplification:

$$
\tanh (\chi)=\sigma(\chi)
$$

Before proceeding with the control design, let us present the following useful properties [26]

- P1. $\sec ^{2} \chi=\left[1-\sigma^{2}(\chi)\right] \leq 1$

- P2. $\dot{\sigma}(\chi)=\dot{\chi} \sec ^{2} \chi=\dot{\chi}\left[1-\sigma^{2}(\chi)\right]$

- P3. $|\sigma(\chi)| \leq|\chi|$

Step 1: Let us propose the Candidate Lyapunov Function (CLF) to deduce a control that stabilizes the first integrator subsystem (9a)

$$
\mathcal{V}_{1}\left(\xi_{1}\right)=\ln \cosh \left(\xi_{1}\right)
$$

whose time-derivative is give by

$$
\dot{\mathcal{V}}_{1}(\xi)=\sigma\left(\xi_{1}\right) \xi_{2}
$$

which is rendered negative-definite $\left(\dot{\mathcal{V}}_{1}(\xi)<0\right)$ if we consider $\xi_{2}$ as virtual (bounded) control input. Thus, we take:

$$
\xi_{2}=-\lambda_{1} \sigma\left(\xi_{1}\right)
$$

where $\lambda_{1}$ is a positive scalar constant. Hence, we can conclude that $\xi_{1}$ is not only stable but also converges asymptotically to the origin.

Step 2: Now, let us propose an error state variable $z$, where the previous bounded virtual controller (see eq. 13) is now used as the reference trajectory, $\xi_{2}^{d}=-\lambda_{1} \sigma\left(\xi_{1}\right)$, which leads to have a bounded $\xi_{2}$ [27]. Thus we have

$$
z=\xi_{2}-\xi_{2}^{d}=\xi_{2}+\lambda_{1} \sigma\left(\xi_{1}\right)
$$

Solving for $\xi_{2}$ in (14) produces the modified state

$$
\xi_{2}=z-\lambda_{1} \sigma\left(\xi_{1}\right)
$$

The time-derivative of (14) is given by

$$
\dot{z}=\dot{\xi}_{2}+\lambda_{1} \sec ^{2}\left(\xi_{1}\right) \xi_{2}
$$

which allows to rewrite the second-order system as

$$
\begin{aligned}
& \dot{\xi}_{1}=\xi_{2} \\
& \dot{z}=\dot{\xi}_{2}+\lambda_{1} \sec ^{2}\left(\xi_{1}\right)\left[\xi_{2}+\lambda_{1} \sigma\left(\xi_{1}\right)\right]
\end{aligned}
$$

where we have used (15) to replace $\xi_{2}$. In order render stable the state trajectories of the system (17) let the final CLF be

$$
\mathcal{V}_{2}\left(\xi_{1}, z\right)=\ln \cosh \left(\xi_{1}\right)+\frac{1}{2} z^{2}+\frac{1}{2 \gamma} \tilde{\theta}^{2}
$$

where

$$
\tilde{\theta}=\hat{\theta}-\theta
$$

represents the estimation error. The corresponding timederivative of $\mathcal{V}_{2}\left(\xi_{1}, z\right)$ is

$$
\dot{\mathcal{V}}_{2}\left(\xi_{1}, z, \tilde{\theta}\right)=-\lambda_{1} \sigma^{2}\left(\xi_{1}\right)+\sigma\left(\xi_{1}\right) z+z \dot{z}+\frac{1}{\gamma} \tilde{\theta} \dot{\tilde{\theta}}
$$

using (17), (19) and P1 in (20) lead to

$$
\begin{aligned}
& \dot{\mathcal{V}}_{2}\left(\xi_{1}, z, \tilde{\theta}\right) \leq \ldots \\
& -\lambda_{1} \sigma^{2}\left(\xi_{1}\right)+\sigma\left(\xi_{1}\right) z+z\left[\dot{\xi}_{2}+\lambda_{1}\left(z-\lambda_{1} \sigma\left(\xi_{1}\right)\right)\right]+\frac{1}{\gamma} \tilde{\theta} \dot{\hat{\theta}}
\end{aligned}
$$

Remind that:

$$
\dot{\xi}_{2}=\frac{1}{a}\left[d_{p} u(t)+\theta-b \dot{\delta}-\delta\right]-\ddot{\delta}^{d}
$$




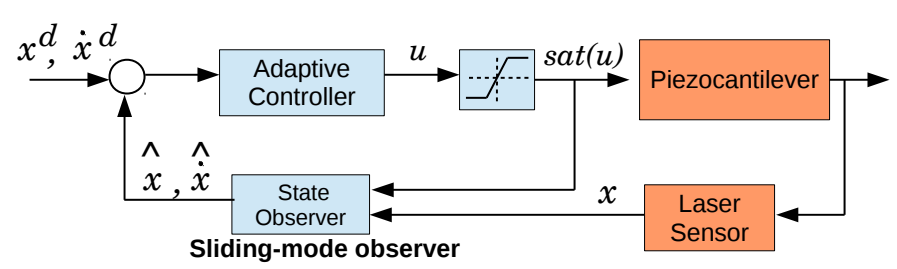

Fig. 2. Block diagram including the observer and the controller.

Therefore, in order to render (21) into a negative function, we introduce the following controller in (22):

$$
\begin{aligned}
& u= \\
& \frac{1}{d_{p}}\left[-\hat{\theta}+a\left(\ddot{\delta}^{d}-\lambda_{1}\left(z+\lambda_{1} \sigma\left(\xi_{1}\right)\right)-\sigma\left(\xi_{1}\right)-\lambda_{2} z\right)\right]
\end{aligned}
$$

Notice that the nominal dynamics of the piezocantilever $(\delta+b \dot{\delta})$ was neglected for the practical implementation of the controller. It can be included in the controller but this implies a prior identification process. Furthermore, using the adaptation law

$$
\dot{\hat{\theta}}=\gamma z
$$

will cancel the term associated to estimation error in (21). The latter leads to

$$
\dot{\mathcal{V}}_{2}\left(\xi_{1}, z, \tilde{\theta}\right)=-\lambda_{1} \sigma^{2}\left(\xi_{1}\right)-\lambda_{2} \sigma^{2}(z)
$$

This result implies that $\dot{\mathcal{V}}_{2}$ is negative-semidefinite $\left(\dot{\mathcal{V}}_{2} \leq\right.$ $0)$. Therefore, it can be only said that $\sigma\left(\xi_{1}\right), \sigma(z)$ and $\theta$ are bounded. Then, the equation (25) can be rewritten as

$$
\dot{\mathcal{V}}_{2}(\mathbf{x}, \tilde{\theta})=-\mathbf{x}^{T} K_{\lambda} \mathbf{x}^{T}
$$

with $\mathbf{x}=\left(\sigma\left(\xi_{1}\right), \sigma(z)\right)^{T}$ and $K_{\lambda}=\operatorname{diag}\left(\lambda_{1}, \lambda_{2}\right)$. The vector

$$
\lim _{t \rightarrow \infty} \mathbf{x} \rightarrow 0
$$

based on the Barbalat's lemma because $\int_{0}^{\infty} \lambda_{\min }\left\{K_{\lambda}\right\}\|\mathbf{x}\|^{2}$ $\langle c$ with $c>0$. However, estimation error can only be guaranteed to be bounded (which was good enough for the experimental tests).

\section{NUMERICAL SIMULATIONS}

The aim of this section is to evaluate the performances of the proposed observer and control approach for the disturbed system in equation (8) at simulation level. Notice that the simulation permits to validate both the observer and the control in details while the experimental tests of the next section only permits to analyze the results of the control. Indeed, there is no sensor to measure the velocity and then to discuss more in details on the efficiency of the observer. For the simulation, a hysteresis model that tracks $h(t)$ of equation (1) is required. For that, we proposes the Bouc-Wen model for which the hysteresis $h(t)$ is given by:

$$
\dot{h}=A_{b w} \dot{u}-B_{b w}|\dot{u}| h-C_{b w} \dot{u}|h|
$$

where $A_{b w}, B_{b w}$ and $C_{b w}$ are parameters that control the shapes of the hysteresis. These parameters have been identified using the least-square algorithm by using experimental hysteresis data from the piezocantilever that will be used for the experiments in the next section. On the other hand, an ARMAX approach (Autoregressivemoving-average model with exogenous inputs) has been employed to identify the parameters of the dynamics part of (8) with an experimental step response of the piezocantilever. We obtain: $a=4.4209 \times 10^{-9}$ and $b=3.7378 \times^{-6}$.

\begin{tabular}{cr}
\hline \hline Parameter & Value[units] \\
\hline \hline$d_{p}$ & $6.8[\mu \mathrm{m} / \mathrm{V}]$ \\
$A_{b w}$ & $3.08[\mu \mathrm{m} / \mathrm{V}]$ \\
$B_{b w}$ & $0.2556\left[V^{-1}\right]$ \\
$C_{b w}$ & $0.0411\left[V^{-1}\right]$ \\
\hline
\end{tabular}

Table 1. Bouc-Wen Model's parameters

Fig.2 depicts the block diagram of the controller and of the observer.

\subsection{Observer performances}

In this case, the state vector is partially known: position is a measured state and velocity is reconstructed. The performances, in open-loop, of the sliding-mode observer are depicted on Fig.3 where it can be observed that estimated position and velocity satisfactorily fulfill the desired performances objective. In particular, Fig.3-c and $\mathrm{d}$ show the quick convergence of the estimate position and of the estimate velocity to the actual position and actual velocity respectively. Indeed, the convergence times for both observation are less than $6 \mathrm{~ms}$.

\subsection{Control performances}

The performances of the controlled piezoelectric cantilever (with observer and controller) while tracking three sine trajectories (amplitude: $50 \mu \mathrm{m}$ and frequency: $1 \mathrm{~Hz}, 10 \mathrm{~Hz}$ and $30 H z$ ) are depicted in figures Fig.4 (for $f=1 H z$ ), Fig.5 (for $f=10 \mathrm{~Hz}$ ) and Fig.6 (for $f=30 \mathrm{~Hz}$ ). From Fig.4-a and c, Fig.5-a and c and Fig.6-a and c, it is seen that that the error for the position tracking is less than $1 \%$ at stationary regime. Concerning the velocity tracking, we see from Fig.4-b and d, Fig.5-b and d and Fig.6-b and $d$ that the error is negligible. To summarize, for low or high frequencies of the reference, the control strategy ensures a satisfactorily behavior although we are using a reconstructed velocity state and that the disturbance was assumed with a slow-time varying dynamics in the
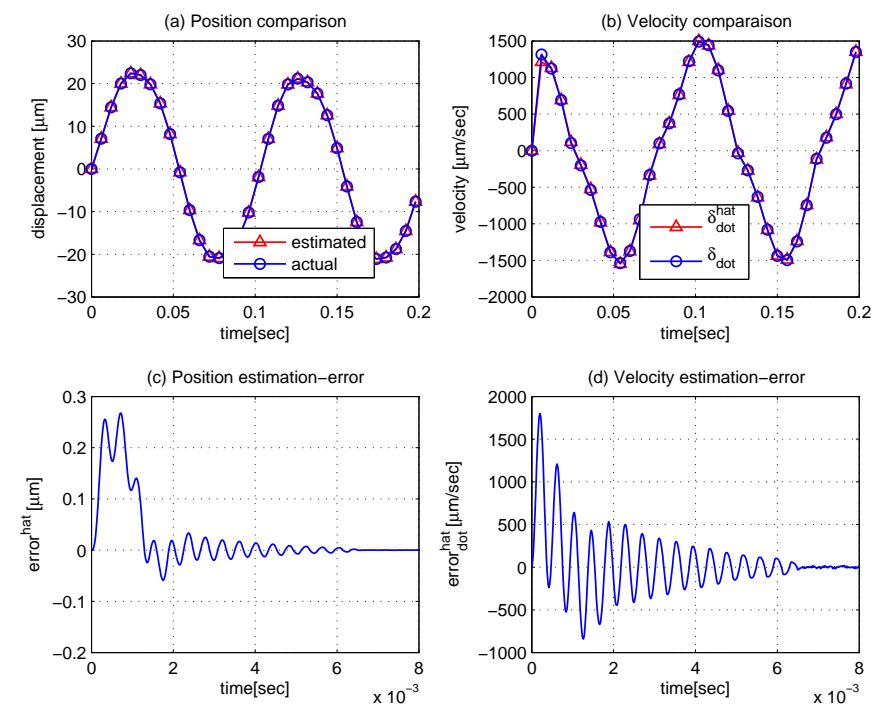

Fig. 3. Observer performances in open loop. (a): actual position and estimate position. (b): actual velocity and estimate velocity. (c): error on position. (d): error on velocity. 

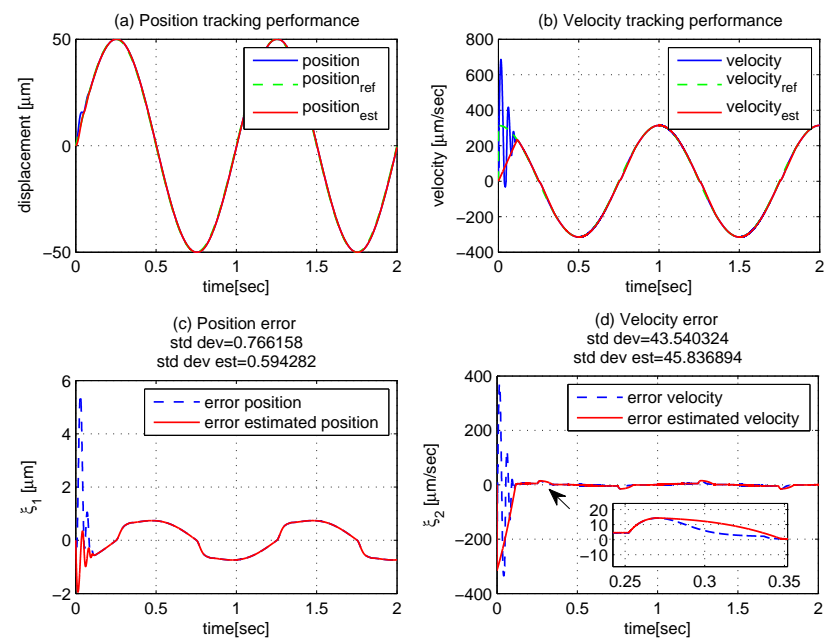

Fig. 4. Trajectory tracking performance @ 1hz
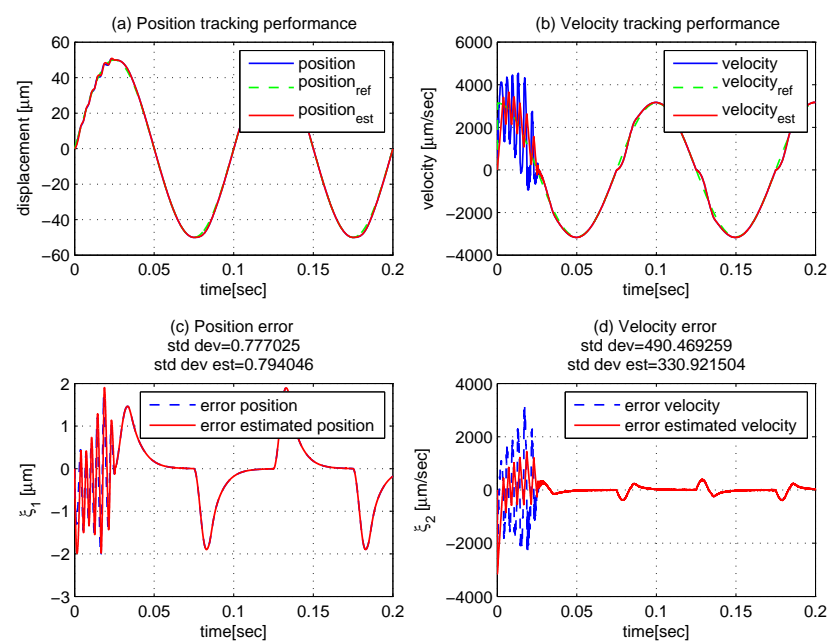

Fig. 5. Trajectory tracking performance @ 10hz

synthesis. The observer gains and control gains used for the numerical simulations are depicted in table 2 . These values were calculated manually in order to satisfy the conditions given in section-3. Recall that the controller gains are multiplied by the dynamic parameter $a=4.4209 \times 10^{-9}$ as depicted in equation (23).

\begin{tabular}{crrr}
\hline Observer gains & $\mathbf{f = 1 H z}$ & $\mathbf{f}=\mathbf{1 0 H z}$ & $\mathbf{f}=\mathbf{3 0 H z}$ \\
\hline$\beta$ & $1.2 \times 10^{2}$ & $20 \times 10^{2}$ & $60 \times 10^{2}$ \\
$\alpha$ & $2 \times 10^{3}$ & $90 \times 10^{4}$ & $580 \times 10^{4}$ \\
\hline Control gains & $\mathbf{f}=\mathbf{1 H z}$ & $\mathbf{f}=\mathbf{1 0 H z}$ & $\mathbf{f}=\mathbf{3 0 H z}$ \\
\hline$\lambda_{1}=\lambda_{2}$ & $15 \times 10^{2}$ & $30 \times 10^{2}$ & $65 \times 10^{2}$ \\
$\gamma$ & 0.3 & 0.3 & 0.4 \\
\hline
\end{tabular}

Table 2. Observer and control gains

\section{EXPERIMENTAL TESTS}

In this section, the previous observer and control strategy are implemented and applied to a piezocantilever as experimental tests. The setup, presented in Fig.7. This is composed of:

- the piezocantilever has 36 layers and have active dimensions (active length $\times$ width $\times$ total thickness) of: $15 \mathrm{~mm} \times 2 \mathrm{~mm} \times 2 \mathrm{~mm}$. All layers are based on
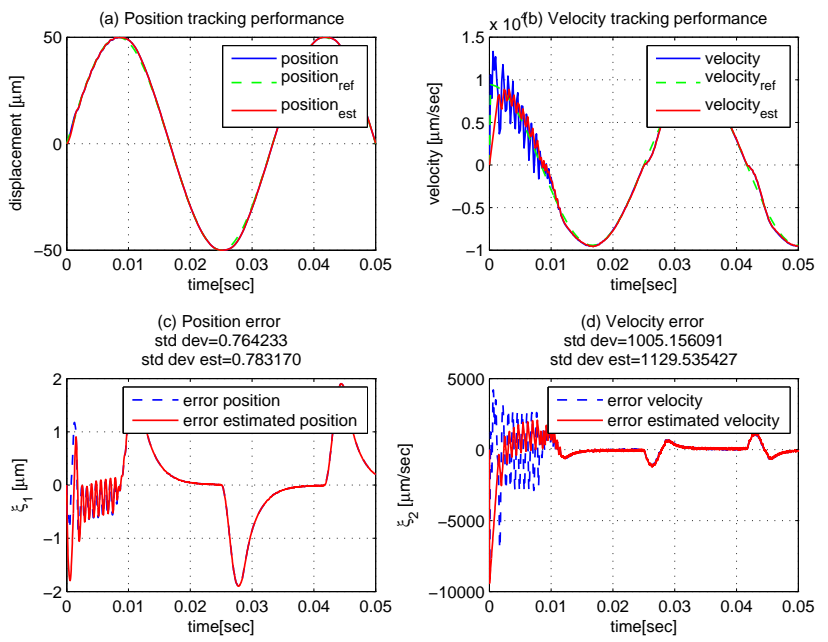

Fig. 6. Trajectory tracking performance @ 30hz

the lead zirconate titanate (PZT) material. This piezocantilever can bends along two directions ( $y$ and $z$ ) but we experiment our observer and control strategy in this paper with the $y$ axis. This bending along $y$ is called $\delta$.

- an optical sensor LC2420 from Keyence Company that is used to measure the bending (position, or displacement) $\delta$ of the tip of the piezocantilever. The sensor is set to have a resolution of $50 \mathrm{~nm}$, an accuracy of $200 \mathrm{~nm}$ and a bandwidth in excess of $1500 \mathrm{kHz}$.

- and a computer and a dSPACE board for the implementation of the observer and the controller and for the management of the different signals. The freshing time is set equal to $0.2 \mathrm{~ms}$ which largely considers the dynamics of the actuator.

The fact that the piezocantilever has several layers permits to work with low voltage (between $-10 \mathrm{~V}$ and $+10 \mathrm{~V}$ ) to obtain sufficient displacement (up to $50 \mu \mathrm{m}$ ). This limitation of the control voltage should be respected, otherwise the electrical field $\frac{u}{e_{p z t}}$ (with $e_{p z t}$ being the thickness of each layer) may be too excessive and results in the depolarization of the layers. The saturation operation considered by the control strategy developed in section-3 is therefore essential for this application. The observer gains and control gains used for the implementation are that of $f=30 \mathrm{~Hz}$ in table 2 .

The first experimental test consists in applying a step input reference $\delta^{d}=50 \mu \mathrm{m}$ to the closed-loop. The step response is pictured in Fig.8. The result indicates a negligible tracking error of the controlled system and a step response about $7.5 \mathrm{~ms}$.

In the second test, a harmonic analysis is carried out. It consists in applying a sine reference input to the closed-loop at different frequencies ranging between $1 \mathrm{~Hz}$ $(6.283 \mathrm{rad} / \mathrm{s})$ and $1 \mathrm{kHz}(6283 \mathrm{rad} / \mathrm{s})$. The resulting magnitude is afterwards plotted (see Fig.9). As demonstrated by the result, the bandwidth of the closed-loop is in excess of $72 \mathrm{~Hz}(450 \mathrm{rad} / \mathrm{s})$ which is higher than that expected in simulation $(30 \mathrm{~Hz})$. 


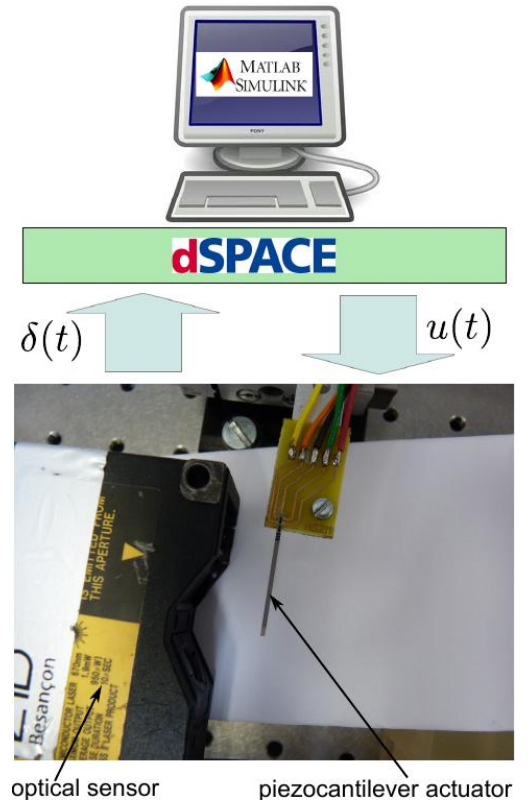

Fig. 7. Presentation of the setup.

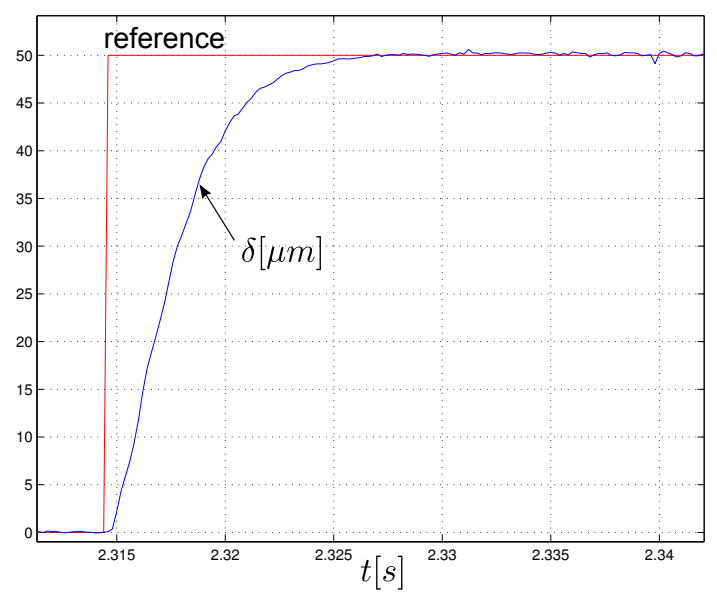

Fig. 8. Experimental step response.

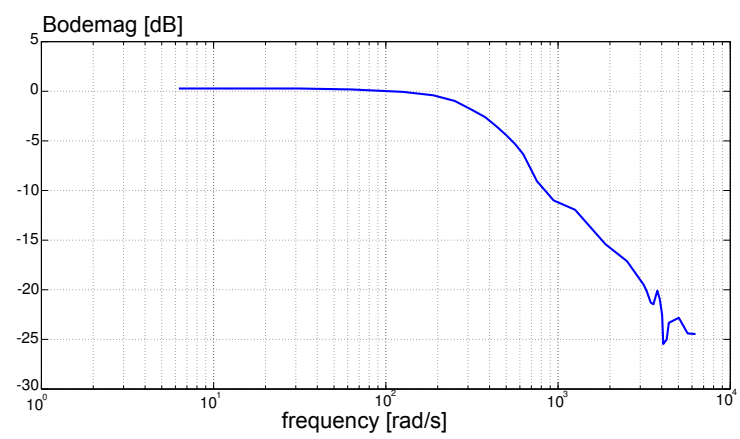

Fig. 9. Experimental harmonic response.

\section{CONCLUDING REMARKS}

In this paper the tracking control problem was addressed for a piezocantilever actuator. To deal with such problem, we have used a sliding-mode observer to estimate the complete state vector of the piezocantilever while having as only measurement the position. The estimated state vector, composed of the position and the velocity, is used in an adaptive control law to counteract the adverse effects provoked by the hysteresis and the creep which are considered as disturbance. Since real-world systems feature a finite response (saturation), we have designed a controller with saturated states via the Backstepping technique considering into account bounded states and bounded virtual controllers throughout the control design. Simulation analysis was first carried out with the observer and control strategy in order to validate the efficiency of the proposed approach. Then experimental tests with a piezocantilever actuator having 36 layers were performed in order to confirm the theory and the simulation results. Both the simulation and the experimental results demonstrated the efficiency and the performances proposed by the observer and the control strategy.

\section{REFERENCES}

[1] D. Hughes and J. T. Wen, "Preisach modeling of piezoceramic and shape memory alloy hysteresis", Smart Materials and Structures, Vol.4, pp.287- 399, 1997.

[2] D. Croft, G. Shed and S. Devasia, "Creep, hysteresis and vibration compensation for piezoactuators: atomic force microscopy application", ASME Journal of Dynamic Systems, Measurement and Control, 2001.

[3] A. Dubra and J. Massa and C.l Paterson, "Preisach classical and nonlinear modeling of hysteresis in piezoceramic deformable mirrors", Optics Express, Vol.13, No 22, pp.9062-9070, 2005.

[4] K. Kuhnen and H. Janocha, "Inverse feedforwrad controller for complex hysteretic nonlinearities in smartmaterials systems", Control of Intelligent System, Vol.29, No 3, 2001.

[5] W. T. Ang, P. K. Kholsa and C. N. Riviere, "Feedforward controller with inverse rate-dependent model for piezoelectric actuators in trajectory-tracking applications", IEEE/ASME Transactions on Mechatronics (Tmech), Vol.12(2), pp.134-142, April 2007.

[6] B. Mokaberi and A. A. G. Requicha, "Compensation of scanner creep and hysteresis for AFM nanomanipulation", IEEE-TASE, Vol.5, No 2, pp.197-208, 2008.

[7] R. Bouc, "Forced vibration of mechanical systems with hysteresis", Conference on Nonlinear Oscillation, Prague, 1967.

[8] Y. K. Wen, "Method for random vibration of hysteresis systems", Journal of the Engineering Mechanics Division, Vol. 102(2), pp.249-263, March/April 1976.

[9] Micky Rakotondrabe, Yassine Haddab and Philippe Lutz, "Modelling and H-inf force control of a nonlinear piezoelectric cantilever", IEEE/RSJ - IROS, (International Conference on Intelligent Robots and Systems), pp:3131-3136, San Diego CA USA, OctNov 2007

[10] Micky Rakotondrabe, Yassine Haddab and Philippe Lutz, "Plurilinear modeling and discrete $\mu$ - synthesis control of a hysteretic and creeped unimorph piezoelectric cantilever", IEEE - ICARCV, (International Conference on Automation, Robotics, Control and Vision), pp:57-64, Grand Hyatt Singapour, December 2006.

[11] Xu, Q. and Li, Y., "Global sliding mode-based tracking control of a piezo-driven xy micropositioning stage 
with unmodeled hysteresis," in The 2009 IEEE/RSJ International Conference on Intelligent Robots and Systems, 755-760 (October 2009).

[12] Shuanghe Yu, Bijan Shirinzadeh, G. A. and Smith, J., "Sliding mode control of a piezoelectric actuator with neural network compensating rate-dependent hysteresis," in [Proceedings of the 2005 IEEE ICRA], 3641-3645 (April 2005).

[13] Chen, X. and Hisayama, T., "Adaptive sliding-mode position control for piezo-actuated stage," IEEE Transactions on Industrial Electronics 55(11), 39273934 (2008).

[14] Liaw, H. C., Shirinzadeh, B., and Smith, J., "Enhanced sliding mode motion tracking control of piezoelectriccactuators," Sensors and Actuators A: Physical 138(1), 194 - 202 (2007).

[15] Y. Haddab, Q. Chen and P. Lutz, "Improvement of strain gauges micro-forces measurement using Kalman optimal filter- ing.", IFAC Mechatronics, 19(4), 2009.

[16] Mohammad Sheikh Sofla, Seyed Mehdi Rezaei, M. Z. M. S., "Hysteresis-observer based robust tracking control of piezoelectric actuators," in [2010 American Control Conference], 4187-4192 (June 2010).

[17] Micky Rakotondrabe and Philippe Lutz, 'Force estimation in a piezoelectric cantilever using the inversedynamics-based UIO technique', IEEE - ICRA, pp:2205-2210, Kobe Japan, May 2009.

[18] Sitti, M., Campolo, D., Yan, J., Fearing, R.S. "Development of PZT and PZN-PT based unimorph actuators for micromechanical flapping mechanisms", IEEE International Conference on Robotics and Automation, ICRA 2001, May 21-26, 2001, Seoul, Korea.

[19] Micky Rakotondrabe, Yassine Haddab and Philippe Lutz, 'Quadrilateral modelling and robust control of a nonlinear piezoelectric cantilever', IEEE - Transactions on Control Systems Technology (T-CST), Vol.17, Issue 3, pp:528-539, May 2009.

[20] Micky Rakotondrabe, "Bouc-Wen modeling and inverse multiplicative structure to compensate hysteresis nonlinearity in piezoelectric actuators", IEEE Transactions on Automation Science and Engineering (T-ASE), Vol.8, Issue.2, pp.428-431, April 2011.

[21] Micky Rakotondrabe, Cedric Clevy and Philippe Lutz, 'Complete open loop control of hysteretic, creeped and oscillating piezoelectric cantilever', IEEE - TASE, Vol.7(3), pp:440-450, July 2010.

[22] J. H. Sussmann, D. E. Sontag, , and Y. Yang, "A general result on the stabilization of linear systems using bounded controls," IEEE Transactions on Automatic Control, vol. 39, no. 12, December 1994.

[23] Teel, "Global stabilisation and restricted tracking for multiple integrators with bounded controls," Systems and Control Letters, vol. 18, pp. 165-171, 1992.

[24] N. Marchand and A. Hably, "Global stabilization of multiple integrators with bounded controls," Automatica, vol. 41, no. 2, pp. 2147-2152, December 2005

[25] J. Davila, "Exact tracking using backstepping control design and high-order sliding modes", IEEE Transactions on Automatic Control, Vol. 58 No. 8, 2013, pp. 2077-2081

[26] R. Kelly, V. Santibanez and A. Loria, "Control of Robot Manipulators in Joint Space", Springer-Verlag
London Limited 2005.

[27] Ngo, K.B. Mahony, R. Zhong-Ping Jiang, "Integrator backstepping design for motion systems with velocity constraint", 5th Asian Control Conference, 2004., Melbourne, Victoria, Australia, 20 